\title{
A SHORT PROOF OF A PRINCIPAL KINEMATIC FORMULA AND EXTENSIONS
}

\author{
W. ROTHER AND M. ZÄHLE
}

\begin{abstract}
Federer's extension of the classical principal kinematic formula of integral geometry to sets with positive reach is proved in a direct way by means of generalised unit normal bundles, associated currents, and the coarea theorem. This enables us to extend the relation to more general sets. At the same time we get a short proof for the well-known variants from convex geometry and differential geometry.
\end{abstract}

\section{INTRODUCTION}

In his famous work [2] Federer extended the principal kinematic formula of Blaschke and Santaló (see, e.g. [5]) to the class of sets with positive reach which contains the convex sets and the $C^{2}$-submanifolds of the euclidean space $R^{d}$ as special cases. In this paper he developed the coarea theorem as a fundamental tool for geometrical integral transformations. Later on Chern [1] gave a more direct proof for the case of $C^{2}$-submanifolds by differential geometric methods.

Our aim is to show that the principal kinematic formula holds for a large class of sets with bounded extrinsic curvature. In the present paper we will derive a short and natural approach to related problems by means of generalised unit normal bundles, associated currents and the coarea theorem in the bundle spaces. Here we will consider only the case of sets with positive reach and certain simple extensions as arbitrary convex surfaces and locally finite unions of these sets.

All details concerning the notions of the next section can be found in Federer [3], and in Zähle [8].

\section{THE PRINCIPAL KINEMATIC FORMULA}

Let $S O(d)$ be the rotation subgroup of the group $G$ of rigid motions in $R^{d}$ and let $\mu$ be the Haar-measure on $G$ whose component $\nu$ on $S O(d)$ is normalised. Furthermore, we denote by $L^{d}$ the $d$-dimensional Lebesgue measure, by $\mathscr{H}^{k}$ the $k$-dimensional Hausdorff (outer) measure, by $B(x, r)$

Received by the editors October 4, 1988 .

1980 Mathematics Subject Classification (1985 Revision). Primary 53C65, 58A25; Secondary $52 \mathrm{~A} 22$. 
the ball with centre $x$ and radius $r$ in $R^{d}, S^{d-1}:=\partial B(0,1)$, and $\beta(d):=$ $\mathscr{H}^{d-1}\left(S^{d-1}\right)$.

Let $X$ be a subset of $R^{d}$ with positive reach and let $a_{X}$ be the unit simple $(d-1)$-vectorfield orienting the unit normal bundle

$$
\text { nor } X:=\left\{(x, n) \in R^{d} \times S^{d-1}: x \in X, n \in \operatorname{Nor}(X, x)\right\}
$$

by

$$
\operatorname{sgn}\left\langle\bigwedge_{d-1}\left(\pi_{0}+t \pi_{1}\right) a_{X}(x, n) \wedge n, e_{1}^{\prime} \wedge \cdots \wedge e_{d}^{\prime}\right\rangle=1,
$$

where $\pi_{0}$ and $\pi_{1}$ are the coordinate mappings with $\pi_{0}(x, n)=x$ and $\pi_{1}(x, n)$ $=n, 0<t<\operatorname{reach} X$, and $e_{1}^{\prime}, \ldots, e_{d}^{\prime}$ is the dual basis of the orthonormal basis $e_{1}, \ldots, e_{d}$ in $R^{d}$.

If we define the $(d-1)$-current $N_{X}$ in $R^{d} \times R^{d}$ (or in $R^{d} \times S^{d-1}$ ) by

$$
N_{X}:=\left(\mathscr{H}^{d-1}\llcorner\operatorname{nor} X) \wedge a_{X}\right.
$$

and the differential forms $\varphi_{k}(0 \leq k \leq d-1)$ in $R^{d} \times R^{d}$ by means of

$$
\left\langle\eta, \varphi_{k}(x, n)\right\rangle:=\beta(d-k)^{-1} \sum_{\substack{\varepsilon_{i}=0,1 \\ \sum \varepsilon_{i}=d-1-k}}\left\langle\pi_{\varepsilon_{1}} \eta_{1} \wedge \cdots \wedge \pi_{\varepsilon_{d-1}} \eta_{d-1} \wedge n, e_{1}^{\prime} \wedge \cdots \wedge e_{d}^{\prime}\right\rangle
$$

if $\eta=\eta_{1} \wedge \cdots \wedge \eta_{d-1} \in \Lambda_{d-1}\left(R^{d} \times R^{d}\right)$ then the $k$ th curvature measure of $X$, $C_{k}(X, \cdot)$ may be expressed by $C_{k}(X, A)=N_{X}\left(1_{A} \varphi_{k}\right)$ for every bounded Borel subset $A$ of $R^{d} \times S^{d-1}, k=0, \ldots, d-1$. Since $\varphi_{k}$ does not depend on $x$, we denote it by $\varphi_{k}(n)$. For completeness we write $C_{d}(X, A):=L^{d}(X \cap A)$.

The principal kinematic formula provides a relation for the curvature measures of the intersection of two sets with positive reach one fixed and the other moving:

Theorem. Suppose $X$ and $Y$ are subsets of $R^{d}$ with reach $X>0$, reach $Y>0$. Then for any bounded Borel sets $A$ and $B$ of $R^{d}$

$$
\int_{G} C_{k}(X \cap g Y, A \cap g B) \mu(d g)=\sum_{i+j=k+d} \gamma(i, j, d) C_{i}(X, A) C_{j}(Y, B)
$$

where

$$
\gamma(i, j, d)=\frac{\Gamma\left(\frac{i+1}{2}\right) \Gamma\left(\frac{j+1}{2}\right)}{\Gamma\left(\frac{i+j-d+1}{2}\right) \Gamma\left(\frac{d+1}{2}\right)}, \quad i+j \geq d,
$$

for the Euler-function $\Gamma$.

Remark. The curvature measures of the intersection sets are determined for $\mu$-a.a. $g \in G$ by the following arguments: According to part 1 of the proof of Theorem 6.11 in [2] (for $r=0$ ) for a.a. $g \in G$ there is no pair $(x, n) \in$ nor $X$ and $(x, m) \in$ nor $g Y$ such that $n=-m$. Therefore in view of Theorem 4.10 in [2] for these $g$, reach $X \cap g Y>0$, provided that one of the sets is 
compact. Thus, the curvature measures in the general case may be introduced by the relation

$$
C_{k}(X \cap g Y, A \cap g B)=N_{X \cap g Y}\left(1_{A \cap g B} \varphi_{k}\right) .
$$

Proof of the theorem. For $k=d$ it is easy to see that

$$
\int_{G} \int_{X \cap g Y} 1_{A \cap g B}(x) \mathscr{C}^{d}(d x) \mu(d g)=\mathscr{H}^{d}(X, A) \mathscr{H}^{d}(Y, B),
$$

i.e., the assertion is true. Here the assumption on positive reach is not needed.

If $k \leq d-1$ we have $C_{k}(X \cap g Y, A \cap g B)=N_{X \cap g Y}\left(1_{A \cap g B} \varphi_{k}\right)$. For $g \in G$ as in the remark, let

$$
\begin{aligned}
& F_{g}^{1}:=\operatorname{nor} X \cap\left(g \stackrel{\circ}{Y} \times S^{d-1}\right) \cap(A \cap g B) \times S^{d-1}, \\
& F_{g}^{2}:=\operatorname{nor} g Y \cap\left(\stackrel{\circ}{X} \times S^{d-1}\right) \cap(A \cap g B) \times S^{d-1}, \\
& F_{g}^{3}:=\left\{(x, u) \in \mathbf{R}^{d} \times S^{d-1}: x \in \partial X \cap A \cap g(\partial Y \cap B),\right. \\
&u \in \operatorname{Nor}(X, x)+\operatorname{Nor}(g Y, x)\} \\
&=\left\{\left(x, \frac{\alpha n+(1-\alpha) m}{|\alpha n+(1-\alpha) m|}\right): x \in A \cap g B,(x, n) \in \operatorname{nor} X,\right. \\
&(x, m) \in \operatorname{nor} g Y, \alpha \in[0,1]\},
\end{aligned}
$$

where $\stackrel{\circ}{X}, \stackrel{\circ}{Y}$ denote the interior of the sets $X$ and $Y$, respectively (cf. Figure 1).

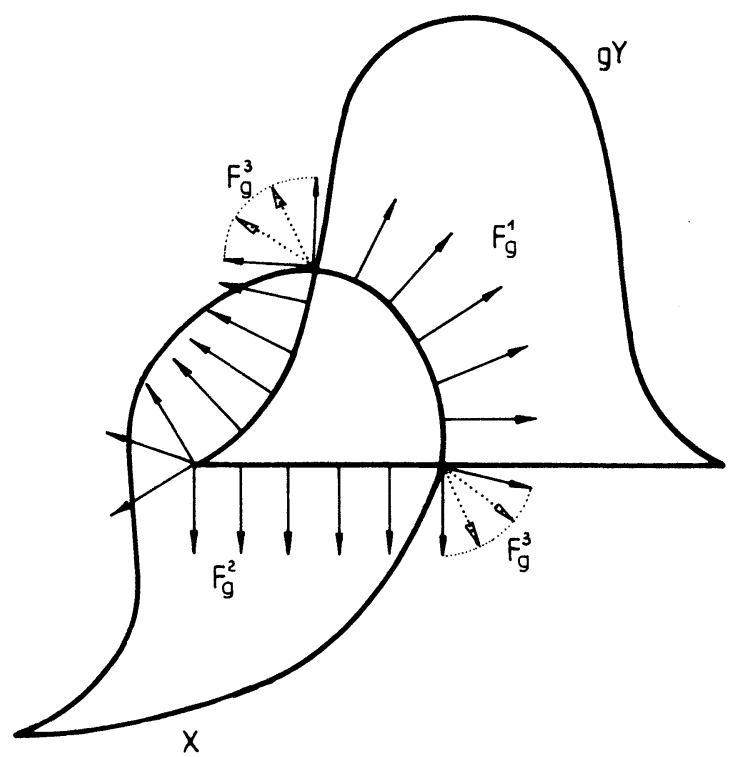

FIGURE 1 
Then $\operatorname{nor}(X \cap g Y) \cap(A \cap g B) \times S^{d-1}$ is the disjoint union of $F_{g}^{1}, F_{g}^{2}$, and $F_{g}^{3}$, and the current may be decomposed:

$$
N_{X \cap g Y}\left\llcorner(A \cap g B) \times S^{d-1}=\left(N_{X \cap g Y}\left\llcorner F_{g}^{1}\right)+\left(N_{X \cap g Y}\left\llcorner F_{g}^{2}\right)+\left(N_{X \cap g Y}\left\llcorner F_{g}^{3}\right) .\right.\right.\right.\right.
$$

The integrals with respect to the Haar-measure $\mu$ on the motion group may be factorized by the translation and the rotation subgroups. Thus we obtain for the first summand

$$
\begin{aligned}
\int_{G}( & \left.N_{X \cap g Y} L F_{g}^{1}\right)\left(\varphi_{k}\right) \mu(d g) \\
= & \int_{G} \int_{F_{g}^{1}} 1_{A \cap g B}(x)\left\langle a_{X \cap g Y}(x, n), \varphi_{k}(n)\right\rangle \mathscr{H}^{d-1}(d(x, n)) \mu(d g) \\
= & \int_{S O(d)} \int_{\mathbf{R}^{d}} \int_{\operatorname{nor} X} 1_{g Y+z}^{\rho_{g}}(x) 1_{A}(x) 1_{g B+z}(x)\left\langle a_{X}(x, n), \varphi_{k}(n)\right\rangle \\
& \times \mathscr{H}^{d-1}(d(x, n)) L^{d}(d z) \nu(d g) \\
= & \int_{S O(d)} \int_{\operatorname{nor} X} 1_{A}(x)\left\langle a_{X}(x, n), \varphi_{k}(n)\right\rangle \mathscr{H}^{d-1}(d(x, n)) \\
& \quad \times \int_{\mathbf{R}^{d}} 1_{Y \cap B}\left(g^{-1} z\right) L^{d}(d z) \nu(d g) \\
= &
\end{aligned}
$$

Analogously, we get

$$
\int_{G}\left(N_{X \cap g Y}\left\llcorner F_{g}^{2}\right)\left(\varphi_{k}\right) \mu(d g)=\mathscr{H}^{d}(X \cap A) C_{k}(Y, B) .\right.
$$

Now it suffices to show that

$$
\int_{G}\left(N_{X \cap g Y}\left\llcorner F_{g}^{3}\right)\left(\varphi_{k}\right)(d g)=\sum_{\substack{i+j=k+d \\ i+j \leq d-1}} \gamma(i, j, d) C_{i}(X, A) C_{j}(Y, B) .\right.
$$

For a fixed rotation $g \in S O(d)$ let

$$
S_{g}:=\left(N_{X} \times N_{g Y} \times[0,1]\right)\left\llcorner\left(A \times S^{d-1} \times g B \times S^{d-1} \times[0,1]\right)\right.
$$

be the restricted cartesian product of $N_{X}, N_{g Y}$ and $[0,1]$. In view of Theorem 3.2.23 of [3] this current is $(2 d-1)$-rectifiable and the corresponding Hausdorffmeasure is a product measure.

We define the following mappings: by

(1) $f:\left(\operatorname{nor} X \cap A \times S^{d-1}\right) \times\left(\operatorname{nor} g Y \cap g B \times S^{d-1}\right) \times[0,1] \rightarrow R^{d} \times R^{d} \times S^{d-1}$

$$
f(x, n, y, m, \alpha)= \begin{cases}\left(x, y, \frac{\alpha n+(1-\alpha) m}{|\alpha n+(1-\alpha) m|}\right), & \alpha n+(1-\alpha) m \neq 0, \\ \left(x, y, e_{1}\right), & \text { otherwise. }\end{cases}
$$

(Let $W_{g}$ be the image space of $f$.) 
(2) $h: W_{g} \rightarrow R^{d}$ by $h(x, y, u)=x-y$.

(3) $p: W_{g} \rightarrow R^{d} \times S^{d-1}$ by $p(x, y, u)=((x+y) / 2, u)$. Note, that for $L^{d} \times \nu$-almost all $(z, g) \in R^{d} \times S O(d)$ we have

$$
\begin{aligned}
p\left(h^{-1}(z) \cap W_{g}\right) & =\left\{\left(x+\frac{z}{2}, \frac{\alpha n+(1-\alpha) m}{|\alpha n+(1-\alpha) m|}\right):(x, n) \in \operatorname{nor} X \cap A \times S^{d-1},\right. \\
& \left.(x+z, m) \in \operatorname{nor} g Y \cap g B \times S^{d-1}\right\} \\
& =\tau_{z / 2} F_{t_{z} \circ g}^{3},
\end{aligned}
$$

where $t_{z}: R^{d} \rightarrow R^{d}, t_{z}(x)=x+z$, and $\tau_{z}: \mathbf{R}^{d} \times S^{d-1} \rightarrow R^{d} \times S^{d-1}, \tau_{z}(x, u)=$ $(x+z, u)$. This is the key for applying Federer's coarea formula (cf. [3, 3.2.22 and the current variant 4.3.8]) to our problem: Let $\Omega:=D e_{1}^{\prime} \wedge \cdots \wedge D e_{d}^{\prime}$ be the volume form in $R^{d}$. Then we have

$$
\begin{aligned}
f_{\#} S_{g} & \left(h^{\#} \Omega \wedge p^{\#} \varphi_{k}\right)=\left(f_{\#} S_{g}\left\llcorner h^{\#} \Omega\right)\left(p^{\#} \varphi_{k}\right)\right. \\
= & \int_{R^{d}}\left\langle f_{\#} S_{g}, h, z\right\rangle\left(p^{\#} \varphi_{k}\right) L^{d}(d z) \\
= & \int_{R^{d}} p_{\#}\left\langle f_{\#} S_{g}, h, z\right\rangle\left(\varphi_{k}\right) L^{d}(d z),
\end{aligned}
$$

where

$$
\langle T, h, z\rangle:=\lim _{r \downarrow 0} \frac{T\left\llcorner h^{\#}\left(1_{B(z, r)} \Omega\right)\right.}{\mathscr{H}^{d}(B(0, r))}
$$

is the slice of the rectifiable current $T$, which exists for $L^{d}$-a.a. $z$. By construction, the current $p_{\#}\left\langle f_{\#} S_{g}, h, z\right\rangle$ is associated with integration over the set $\tau_{z / 2} F_{t_{z} \circ g}^{3}$. Later on we will see that its orienting vectorfield $\omega$ satisfies the relation

$$
\operatorname{sgn}\left\langle\bigwedge_{d-1}\left(\pi_{0}+t \pi_{1}\right) \omega \wedge n, e_{1}^{\prime} \wedge \cdots \wedge e_{d}^{\prime}\right\rangle=(-1)^{d-1}
$$

for sufficiently small $t$. Therefore we obtain for almost all $(z, g) \in R^{d} \times S O(d)$

$$
N_{(X+z / 2) \cap(g Y+z)}\left\llcorner\tau_{z / 2} F_{t_{z} \circ g}^{3}=(-1)^{d-1} p_{\#}\left\langle f_{\#} S_{g}, h, z\right\rangle\right.
$$

and

$$
\begin{aligned}
\int_{G}( & N_{X \cap g Y}\left\llcorner F_{g}^{3}\right)\left(\varphi_{k}\right) \mu(d g) \\
& =2^{-d} \int_{S O(d)} \int_{R^{d}} N_{X \cap(g Y+z / 2)}\left\llcorner F_{t_{z / 2} \circ g}^{3}\left(\varphi_{k}\right) L^{d}(d z) \nu(d g)\right. \\
& =2^{-d} \int_{S O(d)} \int_{R^{d}} N_{(X+z / 2) \cap(g Y+z)}\left\llcorner\tau_{z / 2} F_{t_{z} \circ g}^{3}\left(\varphi_{k}\right) L^{d}(d z) \nu(d g)\right. \\
& =(-1)^{d-1} 2^{-d} \int_{S O(d)} f_{\#} S_{g}\left(h^{\#} \Omega \wedge p^{\#} \varphi_{k}\right) \nu(d g) .
\end{aligned}
$$


By the above formula and the product structure of the Hausdorff-measure on nor $X \times$ nor $g Y \times[0,1]$ the integral on the right-hand side is equal to

$$
\begin{aligned}
& \int_{S O(d)} \int_{\text {nor } X} \int_{\text {nor } Y} \int_{[0,1]} 1_{A}(x) 1_{g B}(y) \\
& \quad \times\left\langle\xi^{g}(x, n, y, m, \alpha), f^{\#}\left(h^{\#} \Omega \wedge p^{\#} \varphi_{k}\right)(x, n, y, m, \alpha)\right\rangle \\
& \times \mathscr{H}^{d-1}(d(x, n)) \mathscr{H}^{d-1}(d(y, m)) L(d \alpha)
\end{aligned}
$$

where $\xi^{g}=\xi_{1}^{g} \wedge \cdots \wedge \xi_{2 d-1}^{g}$ is the orienting vectorfield of nor $X \times$ nor $g Y \times[0,1]$, which is defined as the outer product of the embedded orienting vectorfields of nor $X$, nor $g Y$ and $[0,1]$. In view of the motion invariance of the Hausdorff measure the last expression coincides with

$$
\begin{aligned}
& \int_{S O(d)} \int_{\text {nor } X} \int_{\text {nor } Y} \int_{[0,1]} 1_{A}(x) 1_{B}(y) \\
& \times\left\langle\xi^{g}(x, n, g y, g m, \alpha), f^{\#}\left(h^{\#} \Omega \wedge p^{\#} \varphi_{k}\right)(x, n, g y, g m, \alpha)\right\rangle \\
& \quad \times \mathscr{H}^{d-1}(d(x, n)) \mathscr{H}^{d-1}(d(y, m)) L(d \alpha) .
\end{aligned}
$$

We now will compute the bracket under the integral. (For brevity denote $\xi^{g}$ by $\xi$.)

$$
\begin{aligned}
\left\langle\xi,(h \circ f)^{\#} \Omega \wedge(p \circ f)^{\#} \varphi_{k}\right\rangle & \\
= & \sum_{\sigma \in S h(d, d-1)} \operatorname{sgn} \sigma\left[\bigwedge_{d} D(p \circ f)\left(\xi_{\sigma(1)} \wedge \cdots \wedge \xi_{\sigma(d)}\right)\right] \\
& \times\left\langle\bigwedge_{d-1} D(h \circ f)\left(\xi_{\sigma(d+1)} \wedge \cdots \wedge \xi_{\sigma(2 d-1)}\right), \varphi_{k} \circ h \circ f\right\rangle
\end{aligned}
$$

where for arbitrary $\eta \in \bigwedge_{d} \mathbf{R}^{d},[\eta]:=\left\langle\eta, e_{1}^{\prime} \wedge \cdots \wedge e_{d}^{\prime}\right\rangle$, and for $\left(w_{x}, w_{n}, w_{y}\right.$, $\left.w_{m}, w_{\alpha}\right) \in \operatorname{Tan}(\operatorname{nor} X \times \operatorname{nor} g Y \times[0,1],(x, n, y, m, \alpha))$

$$
\begin{aligned}
& {[D(p \circ f)(x, n, y, m, \alpha)]\left(w_{x}, w_{n}, w_{y}, w_{m}, w_{\alpha}\right)} \\
& =\left(\frac{1}{2}\left(w_{x}+w_{y}\right),\right. \\
& \left.\quad \frac{1}{|\alpha n+(1-\alpha) m|} \Pi_{(\alpha n+(1-\alpha) m)^{\perp}}\left(\alpha w_{n}+(1-\alpha) w_{m}+w_{\alpha}(n-m)\right)\right),
\end{aligned}
$$

$[D(h \circ f)(x, n, y, m, \alpha)]\left(w_{x}, w_{n}, w_{y}, w_{m}, w_{\alpha}\right)=w_{x}-w_{y}$.

In particular, $[D(h \circ f)(x, n, y, m, \alpha)] \xi_{2 d-1}=0$. By definition of $\varphi_{k}$ we get

$$
\begin{aligned}
\left\langle\xi,(h \circ f)^{\#} \Omega \wedge(p \circ f)^{\#} \varphi_{k}\right\rangle & \\
= & \beta(d-k)^{-1} \sum_{\sigma \in S h(d, d-2)} \operatorname{sgn} \sigma\left[\bigwedge_{d} D(h \circ f)\left(\xi_{\sigma(1)} \wedge \cdots \wedge \xi_{\sigma(d)}\right)\right] \\
\times & \sum_{\substack{\varepsilon_{i}=0,1 \\
\varepsilon_{i}=d-1-k}}\left[\Pi_{\varepsilon_{1}} \tilde{\xi}_{\sigma(d+1)} \wedge \cdots \wedge \Pi_{\varepsilon_{d-2}} \tilde{\xi}_{\sigma(2 d-2)} \wedge \Pi_{\varepsilon_{d-1}} \tilde{\xi}_{2 d-1}\right. \\
& \left.\wedge \frac{\alpha n+(1-\alpha) g m}{|\alpha n+(1-\alpha) g m|}\right]
\end{aligned}
$$


where $\tilde{\xi}_{\sigma(i)}:=D(p \circ f) \xi_{\sigma(i)}$ for $i=d+1, \ldots, 2 d-2$, and $\tilde{\xi}_{2 d-1}:=$ $D(p \circ f) \xi_{2 d-1}$. Since $\pi_{0} \tilde{\xi}_{2 d-1}=0$, we have $\left\langle\xi,(h \circ f)^{*} \Omega \wedge(p \circ f)^{*} \varphi_{d-1}\right\rangle=0$, and so the principal kinematic formula is true in the case $k=d-1$.

Hence, we may assume that $k \leq d-2$. Furthermore,

$$
\pi_{1} \hat{\xi}_{2 d-1}=\frac{1}{|\alpha n+(1-\alpha) g m|} \Pi_{(\alpha n+(1-\alpha) g m)^{\perp}}(n-g m),
$$

and the outer product possesses the property that $a \wedge b=a \wedge(b+c a), a, b \in R^{d}$, $c \in R$. Therefore we have

$$
\begin{aligned}
&\left\langle\xi,(h \circ f)^{\#} \Omega \wedge(p \circ f)^{*} \varphi_{k}\right\rangle \\
&= \beta(d-k)^{-1} \sum_{\sigma \in S h(d, d-2)} \operatorname{sgn} \sigma \frac{1}{|\alpha n+(1-\alpha) g m|^{d-1-k}} \\
& \quad \times \sum_{\substack{\varepsilon_{i}=0,1 \\
\varepsilon_{i}=d-2-k}}\left[\lambda_{1} \pi_{\varepsilon_{1}} a_{\sigma(d+1)} \wedge \cdots \wedge \lambda_{d-2} \pi_{\varepsilon_{d-2}} a_{\sigma(2 d-2)}^{g} \wedge n \wedge g m\right],
\end{aligned}
$$

where $a_{1} \wedge \cdots \wedge a_{d-1}$ and $a_{d}^{g} \wedge \cdots \wedge a_{2 d-2}^{g}$ are the $(d-1)$-vectorfields orienting nor $X$ and nor $g Y$, respectively,

$$
\mu_{j}:= \begin{cases}+1 & \text { for } \sigma(j) \leq d-1 \\ -1 & \text { for } \sigma(j)>d-1\end{cases}
$$

and

$$
\lambda_{i}:= \begin{cases}\alpha & \text { for } \sigma(d+i) \leq d-1, \varepsilon_{i}=1, \\ 1-\alpha & \text { for } \sigma(d+i)>d-1, \varepsilon_{i}=1, \\ \frac{1}{2}, & \varepsilon_{i}=0 .\end{cases}
$$

Now it is convenient to use the orthonormal bases of $\operatorname{Tan}(\operatorname{nor} X,(x, n))$ and $\operatorname{Tan}(\operatorname{nor} Y,(y, m))$ introduced in $[7,8]$ : Let

$$
\begin{aligned}
& a_{i}(x, n):=\left(\frac{1}{\sqrt{1+\kappa_{i}^{2}(x, n)}} b_{i}(x, n), \frac{\kappa_{i}(x, n)}{\sqrt{1+\kappa_{i}^{2}(x, n)}} b_{i}(x, n)\right) \\
& \text { for } i=1, \ldots, d-1,
\end{aligned}
$$

and

$$
\begin{array}{r}
a_{j+(d-1)}(y, m):=\left(\frac{1}{\sqrt{1+\tilde{\kappa}_{j}^{2}(y, m)}} \tilde{b}_{j}(y, m), \frac{\tilde{\kappa}_{j}(y, m)}{\sqrt{1+\tilde{\kappa}_{j}^{2}(y, m)}} \tilde{b}_{j}(y, m)\right) \\
\quad \text { for } j=1, \ldots, d-1 .
\end{array}
$$

$\kappa_{i}(x, n)$ and $\tilde{\kappa}_{j}(y, m)$ are the $i$ th and $j$ th generalised principal curvature of $X$ and $Y$ at $x$ and $y$ with respect to the normals $n$ and $m$, respectively, which are defined in [7], 
$\left(b_{i}\right)_{i=1, \ldots, d-1}$ and $\left(\tilde{b}_{j}\right)_{j=1, \ldots, d-1}$ are the orthonormal systems of directions of principal curvatures such that $b_{1} \wedge \cdots \wedge b_{d-1} \wedge n=\tilde{b}_{1} \wedge \cdots \wedge \tilde{b}_{d-1} \wedge m=$ $e_{1} \wedge \cdots \wedge e_{d}$.

Then $a_{g Y}=a_{d}^{g} \wedge \cdots \wedge a_{2 d-2}^{g}$ with $a_{j+(d-1)}^{g}(g y, g m)=\left(\frac{1}{\sqrt{1+\tilde{\kappa}_{j}(y, m)}} g \tilde{b}_{j}(y, m), \frac{\tilde{\kappa}_{j}(y, m)}{\sqrt{1+\tilde{\kappa}_{j}(y, m)}} g \tilde{b}_{j}(y, m)\right)$ for $j=1, \ldots, d-1$, forms an orienting vectorfield of nor $g Y$. Since

$$
\begin{aligned}
& \sum_{\substack{\varepsilon_{i}=0,1 \\
\varepsilon_{i}=d-1-k}} \sum_{\sigma \in \operatorname{Sh}(d, d-2)} \cdots
\end{aligned}
$$

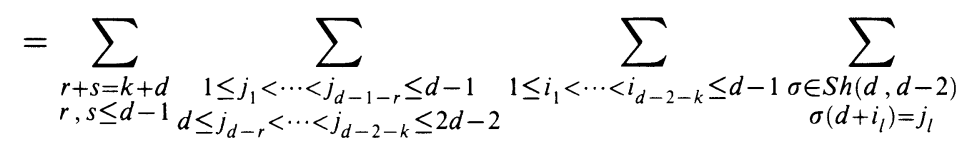

we obtain

$$
\begin{aligned}
& \left\langle\xi,(h \circ f)^{\#} \Omega \wedge(p \circ f)^{\#} \varphi_{k}\right\rangle \\
& =\beta(d-k)^{-1} 2^{-k} \sum_{\substack{r+s=k+d \\
r, s \leq d-1}} \sum_{\substack{1 \leq j_{1}<\cdots<j_{d-1-r} \leq d-1 \\
d \leq j_{d-r}<\cdots<j_{d-2-k} \leq 2 d-2}} \sum_{1 \leq i_{1}<\cdots<i_{d-2-k} \leq d-1} \\
& \times \sum_{\substack{\sigma \in S h(d, d-2) \\
\sigma\left(d+i_{l}\right)=j_{l}}} \operatorname{sgn} \sigma \frac{\alpha^{d-1-r}(1-\alpha)^{d-1-s}}{|\alpha n+(1-\alpha) g m|^{d-1-k}}(-1)^{d-q_{\sigma}} \\
& \times \prod_{t=1}^{d-1} \frac{1}{\sqrt{1+\kappa_{t}^{2}} \sqrt{1+\tilde{\kappa}_{t}^{2}}} \prod_{l_{1}=1}^{d-1-r} \kappa_{l_{l_{1}}} \prod_{l_{2}=d-r}^{d-2-k} \tilde{\kappa}_{l_{l_{2}-(d-1)}} \\
& \times\left[b_{\sigma(1)} \wedge \cdots \wedge b_{\sigma\left(q_{\sigma}\right)} \wedge g \tilde{b}_{\sigma\left(q_{\sigma}+1\right)-(d-1)} \wedge \cdots \wedge g \tilde{b}_{\sigma(d)-(d-1)}\right] \\
& \times\left[b_{\sigma(d+1)} \wedge \cdots \wedge b_{\sigma(2 d-1-q)} \wedge g \tilde{b}_{\sigma\left(2 d-q_{\sigma}\right)-(d-1)}\right. \\
& \left.\wedge \cdots \wedge g \tilde{b}_{\sigma(2 d-2)-(d-1)} \wedge n \wedge g m\right],
\end{aligned}
$$

where $q_{\sigma}=\max \{t: \sigma(t) \leq d-1\}$.

A little linear algebra shows that

$$
\begin{aligned}
{\left[b_{\sigma(d+1)} \wedge \cdots \wedge b_{\sigma\left(2 d-1-q_{\sigma}\right)} \wedge g \tilde{b}_{\sigma\left(2 d-q_{\sigma}\right)-(d-1)} \wedge \cdots \wedge g \tilde{b}_{\sigma(2 d-2)-(d-1)} \wedge n \wedge g m\right] } \\
=(-1)^{q_{\sigma}-1}\left[b_{\sigma(d+1)} \wedge \cdots \wedge b_{\sigma\left(2 d-1-q_{\sigma}\right)} \wedge n \wedge g \tilde{b}_{\sigma\left(2 d-q_{\sigma}\right)-(d-1)}\right. \\
\left.\wedge \cdots \wedge g \tilde{b}_{\sigma(2 d-2)-(d-1)} \wedge g m\right] \\
\operatorname{sgn} \sigma=\left[b_{\sigma(1)} \wedge \cdots \wedge b_{\sigma\left(q_{\sigma}\right)} \wedge b_{\sigma(d+1)} \wedge \cdots \wedge b_{\sigma\left(2 d-1-q_{\sigma}\right)} \wedge n\right] \\
\times\left[\tilde{b}_{\sigma\left(q_{\sigma}+1\right)-(d-1)} \wedge \cdots \wedge \tilde{b}_{\sigma(d)-(d-1)} \wedge \tilde{b}_{\left(2 d-q_{\sigma}\right)-(d-1)}\right. \\
\left.\wedge \cdots \wedge \tilde{b}_{\sigma(2 d-2)-(d-1)} \wedge m\right]
\end{aligned}
$$


and for any two orthonormal bases $v_{1}, \ldots, v_{d}$ and $w_{1}, \ldots, w_{d}$ of $R^{d}$,

$$
\begin{aligned}
{\left[v_{1} \wedge \cdots\right.} & \left.\wedge v_{k} \wedge w_{1} \wedge \cdots \wedge w_{d-k}\right] \\
& =\left[w_{1} \wedge \cdots \wedge w_{d}\right]\left[v_{1} \wedge \cdots \wedge v_{d}\right]\left[v_{k+1} \wedge \cdots \wedge v_{d} \wedge w_{d-k+1} \wedge \cdots \wedge w_{d}\right] .
\end{aligned}
$$

Hence, the above expression equals

$\left\langle\xi,(h \circ f)^{\#} \Omega \wedge(p \circ f)^{\#} \varphi_{k}\right\rangle$

$$
\begin{aligned}
= & \beta(d-k)^{-1} 2^{-k}(-1)^{d-1} \sum_{\substack { r+s=k+d \\
\begin{subarray}{c}{1 \leq j_{1}<\cdots<j_{d-1-r} \leq d-1 \\
d \leq j_{d-r}<\cdots<j_{d-2-k} \leq 2 d-2{ r + s = k + d \\
\begin{subarray} { c } { 1 \leq j _ { 1 } < \cdots < j _ { d - 1 - r } \leq d - 1 \\
d \leq j _ { d - r } < \cdots < j _ { d - 2 - k } \leq 2 d - 2 } }\end{subarray}} \sum_{1 \leq i_{1}<\cdots<i_{d-2-k} \leq d-2} \sum_{\substack{\sigma \in S h(d, d-2) \\
\sigma\left(d+i_{l}\right)=j_{l}}} \frac{\alpha^{d-1-r}(1-\alpha)^{d-1-s}}{\alpha n+\left.(1-\alpha) g m\right|^{d-1-k}} \\
& \times \prod_{t=1}^{d-1} \frac{1}{\sqrt{1+\kappa_{t}^{2}}} \frac{1}{\sqrt{1+\tilde{\kappa}_{t}^{2}}} \prod_{\prod_{1}=1}^{d-1-r} \kappa_{j_{l_{1}}} \prod_{l_{2}=d-r}^{d-2-k} \tilde{\kappa}_{j_{l_{2}}-(d-1)} \\
& \times\left[b_{\sigma(1)} \wedge \cdots \wedge b_{\sigma\left(q_{\sigma}\right)} \wedge g \tilde{b}_{\sigma\left(q_{\sigma}+1\right)-(d-1)} \wedge \cdots \wedge g \tilde{b}_{\sigma(d)-(d-1)}\right]^{2} .
\end{aligned}
$$

It is easy to see that

$$
\begin{aligned}
& \sum_{1 \leq i_{1}<\cdots<1_{d-2-k} \leq d-2} \sum_{\sigma \in S h(d, d-2)} \int_{S O(d)} \int_{0}^{1} \frac{\alpha^{d-1-r}(1-\alpha)^{d-1-s}}{|\alpha n+(1-\alpha) g m|^{d-1-k}} \\
& \times\left[b_{\sigma(1)} \wedge \cdots \wedge b_{\sigma\left(q_{\sigma}\right)} \wedge g \tilde{b}_{\sigma\left(q_{\sigma}+1\right)-(d-1)} \wedge \cdots \wedge g \tilde{b}_{\sigma(d)-(d-1)}\right]^{2} d \sigma \nu(d g)
\end{aligned}
$$

is a constant which only depends on $r, s$ and $d$. We denote it by $c(r, s, d)$.

Further,

$$
\begin{aligned}
\int_{\text {nor } X \times \operatorname{nor} Y} & \sum_{\substack{1 \leq j_{1}<\cdots<j_{d-1-r} \leq d-1 \\
d \leq j_{d-r}<\cdots<j_{d-2-k} \leq 2 d-2}} \\
& \times\left(\prod_{t=1}^{d-1} \frac{1}{\sqrt{1+\kappa_{t}^{2}}} \frac{1}{\sqrt{1+\tilde{\kappa}_{t}^{2}}} \prod_{l_{1}=1}^{d-1-r} \kappa_{l_{1}} \prod_{l_{2}=d-r}^{d-2-k} \tilde{\kappa}_{j_{2}-(d-1)}\right) \\
& \times d\left(\mathscr{H}^{2 d-2} L\left(A \times S^{d-1} \times B \times S^{d-1}\right)\right) \\
= & \sum_{1 \leq j_{1}<\cdots<j_{d-1-r}} \int_{\text {nor } X} \prod_{t_{1}=1}^{d-1} \frac{1}{\sqrt{1+\kappa_{t_{1}}^{2}}} \prod_{l_{1}=1}^{d-1-r} \kappa_{j_{l_{1}}} d\left(\mathscr{H}^{d-1} L A \times S^{d-1}\right) \\
& \times \sum_{1 \leq j_{1}<\cdots<j_{d-1-s} \leq d-1} \int_{\text {nor } Y} \prod_{t_{2}=1}^{d-1} \frac{1}{\sqrt{1+\tilde{\kappa}_{t_{2}}^{2}}} \prod_{l_{2}=1}^{d-1-s} \tilde{\kappa}_{j_{l_{2}}} d\left(\mathscr{H}^{d-1} L B \times S^{d-1}\right) \\
= & \beta(d-r) \beta(d-s) C_{r}(X, A) C_{s}(Y, B) .
\end{aligned}
$$

The last equation follows from the relation $N_{X}\left(1_{A} \varphi_{r}\right)=C_{r}(X, A), N_{Y}\left(1_{B} \varphi_{s}\right)=$ $C_{S}(Y, B)$ by means of the same bases for the orienting vectorfields (see also 
[7]). Consequently, one infers from (1) the kinematic formula

$$
\int_{G}\left(N_{X \cap g Y}\left\llcorner F_{g}^{3}\right)\left(\varphi_{k}\right) \nu(d g)=\sum_{\substack{r+s=k+d \\ r, s \leq d-1}} \gamma(r, s, d) C_{r}(X, A) C_{s}(Y, B)\right.
$$

where $\gamma(r, s, d)=\beta(d-r) \beta(d-s) \beta(d-r+d-s)^{-1} 2^{-(r+s)} c(r, s, d)$.

The explicit calculation of the constant $\gamma(r, s, d)$ may be reduced to the well-known case of Hausdorff measures by the choice of an $r$-dimensional $X$ and an $s$-dimensional $Y$, and $k=r+s-d$ (cf. $[1,2,3])$.

It remains to show that $\operatorname{sgn}\left[\bigwedge_{d-1}\left(\pi_{0}+t \pi_{1}\right) \omega \wedge n\right]=(-1)^{d-1}$. By definition of $\varphi_{k}$ it is easy to verify that for $t \rightarrow 0$

$$
\operatorname{sgn}\left[\bigwedge_{d-1}\left(\pi_{0}+t \pi_{1}\right) \omega \wedge n\right]=\operatorname{sgn}\left\langle\omega, \varphi_{k^{*}}\right\rangle,
$$

where $k^{*}=\max \left\{k:\left\langle\omega, \varphi_{k}\right\rangle \neq 0\right\} \quad\left(k^{*}\right.$ is the number of finite curvatures of $X \cap g Y$ at $(x, n))$.

Since $\operatorname{sgn}\left\langle\omega, \varphi_{k^{*}}\right\rangle=\operatorname{sgn}\left\langle\xi,(h \circ f)^{\#} \Omega \wedge(p \circ f)^{\#} \varphi_{k^{*}}\right\rangle$ and $k^{*}=r^{*}+s^{*}-d$, where $r^{*}$ and $s^{*}$ are the numbers of the finite curvatures of $X$ and $Y$ at the corresponding points and normals, we have

$$
\operatorname{sgn}\left\langle\omega, \varphi_{k^{*}}\right\rangle=(-1)^{d-1} \operatorname{sgn}\left(\prod_{t=1}^{d-1} \frac{1}{\sqrt{1+\kappa_{t}^{2}}} \frac{1}{\sqrt{1+\tilde{\kappa}_{t}^{2}}} \prod_{i=1}^{d-1-r^{*}} \kappa_{j_{i}^{*}}^{d-1-\prod^{*}} \prod_{l=1}^{d-\tilde{\kappa}_{j_{l}^{*}}}\right) .
$$

Here $\kappa_{j_{1}^{*}}, \ldots, \kappa_{j_{d-1-r}^{*}}$ and $\tilde{\kappa}_{j_{1}^{*}}, \ldots, \tilde{\kappa}_{j_{d-1-s}^{*}}$ are the infinite curvatures of $X$ and $Y$. (By definition $\kappa_{j}^{*} / \sqrt{1+\kappa_{j^{*}}^{2}}=1$.) Hence, the last expression equals $(-1)^{d-1}$.

\section{EXTENSIONS}

Instead of sets of positive reach we next consider the class $U_{P R}$ of locally finite unions $X=\bigcup_{i=1}^{\infty} X_{i}$, where reach $\bigcap_{i \in I} X_{i}>0$ for any finite index set $I$. In $[6,8]$ the unique additive extension of Federer's curvature measures to $U_{P R}$ is given under some additional condition, and in [9] arbitrary elements of $U_{P R}$ and more general sets are treated. In view of the additivity property

$$
C_{k}(X \cup Y, \cdot)=C_{k}(X, \cdot)+C_{k}(Y, \cdot)-C_{k}(X \cap Y, \cdot)
$$

provided $X, Y, X \cup Y$, and $X \cap Y \in U_{P R}$, the principal kinematic formula for $U_{P R}$-sets may be reduced to the case of sets with positive reach (cf. [6]). The methods of $\S 1$ also admit a direct proof. The main idea will be demonstrated here on the example of convex surfaces or of the closure of the complement of convex sets in $R^{d}$. These types of sets, in general, do not belong to $U_{P R}$, but to the more general class $S R$ of second order rectifiable sets as introduced in [9]. We conjecture that $S R$ is the largest class of subsets of $R^{d}$, for which the principal kinematic formula in the form of $\S 1$ holds. It suffices to consider convex surfaces, since the arguments for the closure of the complement of 
convex sets are similar. By a convex surface $\mathscr{S}_{p}$ we mean the closure of a $p$ dimensional submanifold of $R^{d}$ with or without boundary, which is contained in the boundary of a convex set in some $(p+1)$-dimensional plane of $\mathbf{R}^{d}$, and whose boundary possesses the same property (where $p$ is replaced by $p-1$ ).

The curvature measures for these sets and, generally, for arbitrary $X \in S R$ may be defined as follows: For $x \in X, n \in S^{d-1}$ the Schneider index of $X$ is given by

$$
i_{X}(x, n):=1-\lim _{r \rightarrow 0} \lim _{s \rightarrow 0} \chi(X \cap B(x+(r+s) n, r))
$$

where $\chi$ denotes the Euler-Poincare characteristic in the sense of singular homology $(\chi(\varnothing):=0)$. Then the generalised unit normal bundle of $X$ is determined by

$$
\text { nor } X:=\left\{(x, n) \in X \times S^{d-1}: i_{X}(x, n) \neq 0\right\} .
$$

It is locally $\left(\mathscr{H}^{d-1}, d-1\right)$-rectifiable in the sense of [3]. This enables us to introduce the associated current and curvature measures similarly as in $\S 1$. By means of the index function possible multiplicities and changes of orientation are regarded in an appropriate way:

Definitions. $N_{X}(\varphi):=\int_{\operatorname{nor} X}\left\langle i_{X} a_{X}, \varphi\right\rangle d \mathscr{H}^{d-1}$. is the current associated with the generalised unit normal bundle, where the orienting $(d-1)$-vectorfield $a_{X}$ is defined as before.

$$
C_{k}(X, B):=N_{X}\left(1_{B} \varphi_{k}\right), \quad k=0, \ldots, d-1,
$$

is the $k$ th curvature measure of $X \in S R$.

We now return to the case $X=\mathscr{S}_{p}$. For all $x \in \mathscr{S}_{p}$ and almost all unit normals of $\mathscr{S}_{p}$ at $x$ the set $\mathscr{S}_{p} \cap B(x+(\varepsilon+\delta) n, \delta)$ for small $\varepsilon$ and $\delta$ is topologically equivalent to the truncated boundary of a $(j+1)$-dimensional convex cone, i.e. it is homotopy equivalent to a $(j-1)$-dimensional sphere (which is the empty set if $j=0) . j$ depends on $\mathscr{S}_{p}$ and on $(x, n)$. We also write $j=j_{\mathscr{S}_{p}}(x, n)$. Hence, in this case $i_{\mathscr{S}_{p}}(x, n)=1-\chi\left(S^{j-1}\right)=(-1)^{j}$.

Now suppose that $\mathscr{S}_{p}$ and $\mathscr{S}_{p}$ are two convex surfaces and $p+q \geq d$. Then for $\mu$-a.a. $g \in G$ the set $\mathscr{S}_{p} \cap g \mathscr{S}_{q}$ is a $(p+q-d)$-dimensional surface which belongs to $S R$. It is not necessarily convex, but the index function $i_{\mathscr{P}_{p} \cap g \mathscr{S}_{q}}$ may be computed analogously. Moreover, for $\mu$-a.a. $g \in G$ and $\mathscr{H}^{d-1}$-a.a. $(x, u) \in \operatorname{nor}\left(\mathscr{S}_{p} \cap g \mathscr{S}_{q}\right)$ we have

$$
j_{\mathscr{S}_{p} \cap g \mathscr{S}_{q}}(x, u)=j_{\mathscr{S}_{p}}(x, n)+j_{g \mathscr{S}_{q}}(x, m)
$$

provided that

$$
u=\frac{\alpha n+(1-\alpha) m}{|\alpha n+(1-\alpha) m|}
$$

(where $\alpha, n, m$ are uniquely determined). This follows from the construction of the mappings in Part 1 which also works in the present case. The only 
difference in the proof of the principal kinematic formula consists in regarding the following immediate consequence for the index functions:

$$
i_{\mathscr{S}_{p} \cap g \mathscr{S}_{q}}(x, u)=i_{\mathscr{S}_{p}}(x, n) i_{g \mathscr{S}_{q}}(x, m)
$$

if $g, x, u, n, m$ are as above. The same methods may be applied when both of the sets are of the form $X=\operatorname{closure}(K \backslash L)$ where $L \subset K$ are convex sets. By additivity, sets of the type $X=\operatorname{closure}\left(K \backslash \bigcup_{l=1}^{k} L_{l}\right), L_{l} \subset K$ all convex, are also allowed. Moreover, we may choose all mixed variants, where the sets $X$ and $Y$ are of different types (including sets of positive reach). If the sum of the dimensions of $X$ and $Y$ is less than $d$, then both sides of this formal principal kinematic formula vanish.

Let $U$ be the class of locally finite unions $X=\bigcup_{l=1}^{\infty} X_{l}$ such that arbitrary finite intersections of the sets $X_{l}$, in particular the $X_{l}$ themselves, are of the types considered until now. Then additivity of the curvature measures yields the following.

Theorem 2. The assertion of Theorem 1 remains valid if $X$ and $Y$ are elements of $U$.

Remark. Instead of euclidean space $R^{d}$ we may consider arbitrary spaces of constant curvature. All notions of the present paper may be defined similarly, and the principal kinematic formula holds in the same form. The difference between the spaces appears if the kinematic relation is formulated in terms of the Euler number of the intersection sets as usual in convex and in differential geometry.

At the same time when we developed our measure geometrical approach to this problem, J. H. G. Fu [4] worked out an abstract algebraic variant of the principal kinematic formula, which may also be applied to the case of sets with positive reach.

\section{REFERENCES}

1. S.-S. Chern, On the kinematic formula in integral geometry, J. Math. Mech. 16 (1966), 101-118.

2. H. Federer, Curvature measure, Trans. Amer. Math. Soc. 93 (1959), 418-491.

3. __ Geometric measure theory, Springer, Berlin, 1969.

4. J. H. G. Fu, Kinematic formulas in integral geometry (to appear).

5. L. A. Santaló, Integral geometry and geometric probability, Encyclopedia Math. Appl., vol. 1, Addison-Wesley, 1976.

6. M. Zähle, Curvature measures and random sets I, Math. Nachr. 119 (1984), 327-339.

7. _ Integral and current representation of Federer's curvature measures, Arch. Math. $\mathbf{4 6}$ (1986), 557-567.

8. _ Curvatures and currents for unions of sets with positive reach, Geom. Dedicata 23 (1987), 155-171.

9. __ Normal cycles and second order rectifiable sets (submitted). 